Clio. Femmes, Genre, Histoire

9 | 1999

Femmes du Maghreb

\title{
Images et représentations de la femme dans les contes marocains du Nord-Ouest
}

\section{Leila Messaoudi}

\section{(2) OpenEdition}

1 Journals

\section{Édition électronique}

URL : https://journals.openedition.org/clio/291

DOI : 10.4000/clio.291

ISSN : 1777-5299

Éditeur

Belin

Édition imprimée

Date de publication : 1 avril 1999

ISBN : 2-85816-461-4

ISSN : $1252-7017$

\section{Référence électronique}

Leila Messaoudi, «Images et représentations de la femme dans les contes marocains du NordOuest », Clio. Histoire, femmes et sociétés [En ligne], 9 | 1999, mis en ligne le 01 avril 2005, consulté le 23 avril 2022. URL : http://journals.openedition.org/clio/291 ; DOI : https://doi.org/10.4000/clio.291

Ce document a été généré automatiquement le 23 avril 2022.

Tous droits réservés 


\title{
Images et représentations de la femme dans les contes marocains du Nord-Ouest
}

\author{
Leila Messaoudi
}

1 Relais de valeurs universelles et pérennes, les contes ont de tout temps défié les frontières. Grands voyageurs, ils n'ont cessé d'investir l'espace réel et imaginaire, et de s'imprégner de colorations spécifiques, tributaires des lieux et des groupes sociaux dans lesquels ils sont transmis. Mais au-delà des aspects universels qui les caractérisent, ils sont les médiateurs privilégiés des différentes cultures et de leurs contenus symboliques. Notre hypothèse est que les contes fonctionnent comme des messagers de cultures et de représentations particulières. Ces récits d'origine orpheline, se reproduisent sans peine dans les contextes sociaux les plus variés et s'en trouvent marqués et parfois même conditionnés.

2 Le champ d'analyse est immense, mais nous n'aborderons ici qu'un seul point, celui des représentations relatives à la femme. Représentations dont les supports sont en priorité les personnages féminins, leurs statuts et leurs rôles. Ce sont ces deux aspects que nous voulons présenter brièvement à partir de l'analyse d'un corpus d'une trentaine de contes, recueillis pour la plupart dans le Nord-Ouest du Maroc, entre 1990 et 1997, et dont on trouvera la liste en annexe.

\section{Statuts des personnages féminins et relations de rôles}

3 Malgré leur forte présence et l'importance qui leur est accordée, les personnages féminins n'acquièrent un statut qu'à travers la consanguinité ou l'alliance : les femmes sont filles de, sœurs de, épouses de, co-épouses de, mères de, marâtres de, etc. En dehors de ces rôles, les femmes sont réduites à être des esclaves, des suivantes, etc.

4 Leur identité sociale est rarement définie par l'exercice d'un métier. Les femmes dans les contes que nous avons recueillis ne sont ni couturières, ni brodeuses, ni tisserandes, etc. Pourtant ces métiers ont toujours existé, mais ils n'apparaissent pas, peut-être 
parce qu'ils sont peu reconnus. Seules les tâches ménagères sont évoquées : laver le linge, nettoyer le sol, faire la vaisselle, la corvée de bois ou la corvée d'eau, arroser le basilic, etc. La cuisine occupe aussi une part importante. D'ailleurs les noms des plats cités permettent d'identifier la région d'appartenance du conteur et le lieu où le conte a été relayé. Ainsi les rôles féminins se définissent par les tâches domestiques qui sont effectuées dans un espace délimité, celui de la maison et ses environs comme la fontaine pour la corvée d'eau et la forêt pour la corvée de bois. Viennent ensuite les autres "fonctions": celles de se marier pour exister socialement et d'enfanter un garçon pour s'affirmer et affronter sa belle famille et ses coépouses. Combien de drames sont fomentés, provoqués par des coépouses jalouses et n'ayant enfanté que des filles! L'univers des contes relègue la femme aux seules tâches traditionnelles et les portraits présentés insistent sur le degré d'habileté à les exécuter.

Rares sont les contes où la femme est louée pour son esprit ou son intelligence. Même la vieille ammeti ou mmi Settoute (tante ou mère Settoute), la vieille femme "petite, maigre, frustrée et rusée » des contes berbères étudiés par Tassadit Yacine, n'apparaît dans les contes marocains que fossilisée dans des formules figées qui servent de prélude! Seul le conte de L'arroseuse du basilic fait exception. L'héroïne parvient à déchiffrer des énigmes qui lui permettent de conquérir le cœur du prince. Toutefois, cette intelligence n'est pas reconnue pour elle-même mais dans la mesure où elle conduit au but de séduire et d'épouser le prince.

6 L'esprit de saillie, la dextérité et la pertinence dans les propos ne sont valorisés que dans la mesure où ils contribuent à la séduction du prince (comme dans L'arroseuse $d u$ basilic) ou à la défense d'un père (comme dans lfahma). En revanche les contes qui relatent les ruses d'un personnage masculin comme dans Hdiddane ou qui louent l'esprit d'un héros (comme dans Le devin sage), ont pour finalité la mise en valeur de cette intelligence comme telle et non pour l'attrait qu'elle pourrait exercer sur un cœur féminin!

7 Les contes révèlent des images archaïques de la femme et ne s'en écartent presque jamais. Curieusement, ils ne se renouvellent pas et les contes sur la polygamie persistent encore sous de multiples versions ! Une violence insoupçonnée sévit dans ces récits où le pire ennemi de la femme est la femme! Ceci nous conduit à traiter des aspects relationnels des personnages féminins. Les relations de rôles ne sont jamais de type transactionnel vu que les personnages féminins n'exercent jamais un métier ou une haute fonction. Elles sont plutôt de type personnel. C'est dans ce cadre que se construisent et se déconstruisent les relations.

\section{Des femmes contre des femmes}

8 Nous ne reprendrons pas le titre Des mères contre les femmes de C. Lacoste-Dujardin. Il ne s'agit pas seulement de mères mais surtout de femmes qui se mènent une guerre sans merci. L'on est profondément choqué à l'écoute de ces violences. Les devoirs et droits fondamentaux sont oubliés. Les principes moraux sont quasi-inexistants chez ces personnages féminins déchaînés.

Prenons quelques exemples:

La coépouse qui boudait la fête du baptême, profita de l'obscurité de la nuit quand tout le monde dormait, pour couper un doigt du petit, couvrir les lèvres de la mère 
de sang et aller porter la fâcheuse nouvelle au mari, en lui faisant croire que la mère a dévoré son nourrisson (La femme qui a mangé son bébé)

... les trois frères se marièrent et habitèrent la même maison avec leur soeur unique et orpheline Les épouses n'apprécièrent guère cette cohabitation. Elles pensèrent à un moyen de faire éloigner leur belle sœur. Elles cherchèrent des œufs de serpent qu'elles lui donnèrent à manger. Aussitôt qu'elle les mangea, son ventre se gonfla. Elles firent croire aux époux que leur sœur avait commis une faute et qu'elle était enceinte. Les trois frères se réunirent et décidèrent de l'éloigner de la maison et chargèrent le plus jeune d'entre eux de la tuer (Wajda l'orpheline)

Il était une fois deux demi-sœurs qui vivaient tranquillement avec leurs parents jusqu'au jour où la mère de l'une d'elles mourut. Dès lors, la vie de la pauvre orpheline se transforma. Elle devint le souffre-douleur de sa belle-mère. Sa demisœur était choyée. Quant à elle, elle était maltraitée par la marâtre qui la chargeait d'accomplir toutes les tâches ménagères et de trimer de l'aube au coucher du soleil. On finit par la surnommer « Aicha des cendres » car elle était tout le temps fourrée dans la cuisine, près des cendres (Aicha des cendres ou Marirda des cendres pour la version berbère.) [N.B. - Ces deux versions aussi bien arabe que berbère sont des variantes marocaines de Cendrillon]

Des exemples de ce genre prolifèrent dans les récits racontés par les femmes et nous ne pouvons les citer tous dans le cadre restreint de cette étude. Des haines exacerbées, des trahisons infâmes, des jalousies corrosives animent ces personnages féminins dont le seul pôle d'intérêt, le «seul objet de convoitise " est la place qui leur est accordée symboliquement par l'homme. Être la préférée de l'époux, du père, du maitre semble être leur seule préoccupation. Elles ne tirent leur raison d'être que de cette place privilégiée et œuvrent par tous les moyens illicites et amoraux pour y parvenir. Ainsi la femme est la première à transgresser les principes moraux lorsque son existence symbolique, liée à la reconnaissance par l'homme, se trouve menacée. La menace est souvent incarnée par une autre femme qui devient l'ennemie. En revanche et paradoxalement, la femme qui transgresse souvent les principes moraux est aussi la gardienne de la bonne moralité du groupe social: le conte de Hina en est un bon exemple.

Youssef trouva en se promenant une belle mèche de cheveux, longue, très longue... Surpris par une mèche aussi belle et aussi longue, il se précipita chez sa mère pour la lui montrer et lui annoncer sa décision: il épouserait la jeune fille à qui appartenait cette mèche fût elle sa propre sœur ! La mère convia les jeunes filles du village et essaya la mèche sur elles. Elle n'appartenait à aucune d'elles. La seule jeune fille qui restait était Hina, la sœur de Youssef. N'ayant pas le choix, la mère essaya la mèche sur sa fille : elle appartenait bien à Hina. Le frère apprenant la nouvelle persista dans sa décision. (...) Hina prit la fuite et s'isola sur un rocher afin d'éviter ce mariage » (Youssef et Hina)

11 Ainsi le mariage incestueux entre le frère et la sœur est évité grâce à la diligence et à la délicatesse de Hina. En effet, si beaucoup de principes sont bafoués, il en est un qui n'est jamais transgressé, celui de l'interdit social qui touche à la structure de la parenté. Sacré, il n'est jamais enfreint dans l'imaginaire des femmes. Dans d'autres versions de ce conte, des femmes conteuses occultent complètement l'inceste. Le frère est remplacé par un cousin, alliance autorisée, mais le mariage est entravé par un ogre.

12 Ainsi la femme est à la source du "bien » social, dans la mesure où elle préserve la cohésion du groupe et protège ses valeurs fondamentales. Elle s'érige en gardienne des coutumes puisque tous les subterfuges qu'elle utilise pour se débarrasser d'une rivale, d'une coépouse etc, n'ébranlent apparemment pas l'édifice solide de la tradition. Le meilleur exemple est celui de la polygamie qui n'est jamais remise en question 
directement. Toutefois celle-ci est fragilisée par les luttes intestines que se livrent les coépouses qui manifestent ainsi leur mécontentement. En fait, les contes, écho du social et de ses normes, sont aussi un prétexte pour sa remise en cause implicite. Lorsque ces femmes s'en prennent à d'autres femmes, c'est généralement à la polygamie qu'elles s'en prennent. C'est aussi contre la discrimination traditionnelle abusive entre un nouveau-né et une «nouvelle-née » qu'elles s'élèvent. Cette violence dirigée en surface contre d'autres femmes s'explique par le fait que ces dernières participent à une structure d'oppression et la confortent. N'ayant pas le pouvoir de transformer des traditions séculaires ancrées dans les mentalités, les femmes se contentent d'éliminer symboliquement, parmi leurs semblables, celles qui acceptent les situations discriminatoires. Et c'est ce thème constant qui traverse une majeure partie des contes marocains, qu'ils soient du Nord ou du Sud, qu'ils soient recueillis en arabe ou en berbère. Cette violence à l'adresse des autres femmes serait à réinterpréter comme une révolte et un refus de telles situations. N'est-ce pas là aussi une revanche sur le réel?

Revanche symbolique, certes, car à aucun moment elle ne touche aux normes du groupe social, sinon de façon indirecte. Comme le note Tassadit Yacine : «Les femmes n'agissent pas ainsi pour défendre leur image de marque mais plutôt l'intérêt du groupe qui leur permet d'exister, fût-ce en marge $»^{1}$. Cette confrontation femmes contre femmes est une compétition pour le droit à l'existence mais elle est aussi le signe d'une révolte et non d'une misogynie ou d'une soumission aux structures. Les femmes sont conservatrices de traditions qu'elles condamnent au fond d'elles-mêmes. C'est ce qui provoque ces images ambivalentes de la femme, telles qu'elles sont véhiculées dans l'imaginaire populaire exprimé par les contes. Elles sont tantôt le signe du bien, tantôt le signe du mal. Et les relations de rôles esquissées illustrent ce dicton marocain :

à la source du bien, une femme

à la source du mal, une femme.

L'image archaïque des femmes offerte par les contes trouve sa contrepartie dans cette révolte exacerbée manifestée par la violence des femmes contre des femmes. Pas n'importe lesquelles! Les actes méchants sont souvent exécutés contre celles qui participent d'une structure profondément refusée de façon inavouée; particulièrement celle de la polygamie. Signe symboliquement précurseur de tous ces mouvements féminins actuels et de toutes ces associations qui revendiquent les droits des femmes à disposer d'elles-mêmes, à exister en tant que telles.

\section{BIBLIOGRAPHIE}

FISHMAN J., 1971, Sociolinguistique, Bruxelles/Paris, Labor/Nathan.

GOROG V. , 1984, «Conte et identité sociale », Cahiers de littérature orale, nº 14. 
LACOSTE-DUJARDIN C., 1985, Des mères contre les femmes. Matriarcat et patriarcat au Maghreb, Paris, La Découverte.

MESSAOUDI L., 1996, « Le conte marocain. Aspects sociolinguistiques de la matière racontée », Recherches pédagogiques, $n^{\circ}$ 6, Meknes, Association Marocaine des Enseignants de Français (AMAEF).

YACINE T., 1993, « la féminité ou la représentation de la peur dans l'imaginaire social kabyle », Cahiers de littérature orale, $\mathrm{n}^{\circ} 34$.

\section{ANNEXES}

Ce corpus est constitué de contes dont les titres sont présentés dans le tableau cidessous. Ils ont été recueillis par des étudiants dans le cadre des activités du GREC (Groupe de Recherches et d'Etudes sur le Conte).

\begin{tabular}{|c|c|}
\hline Lieu de recueil & Titre \\
\hline Kénitra Kén1 & - Sbec bnat (les sept filles) \\
\hline Kén2 & - Hniya, snisla dehbun (Hnia, chaîne dorée) \\
\hline Kén3 & - lbniyya ssRira u xulxalha (la petite fille et son anneau de pied) \\
\hline Sidi Kacem Kac1 & - lmcayza (la petite chèvre) \\
\hline Kac2 & - lmra lli klat ulidha (la mère qui a mangé son fils) \\
\hline Kac3 & - IRula u sebca dlebnat (l'ogresse et les sept filles) \\
\hline Sidi Slimane Sli1 & - lme $\Omega$ fiyya (la jarre) \\
\hline Sli 2 & - -ayat nnufus (Hayat Noufous) \\
\hline Sli 3 & - $\Omega$ diddan l Érami (Hdiddane le rusé) \\
\hline Belksiri Bel 1 & - hayna (Haïna) \\
\hline Bel 2 & - Wajda litima (Wajda l'orpheline) \\
\hline Ouezzane Oue1 & - $\Omega$ diddan u lRula (Hdiddane et l'ogresse) \\
\hline Oue2 & - lunja (Lounja) \\
\hline Oue3 & - lcate llah (Dieu donateur) \\
\hline Oue4 & - ssoltan (Le sultan) \\
\hline Oue5 & - Sidi jjuhar f cnaqdu (Sidi Jouhar fi ‘naqdu) \\
\hline Sidi Redouane Red1 & - lmcaza zzuRbiya (la chèvre maudite) \\
\hline
\end{tabular}




\begin{tabular}{|c|c|}
\hline Lhait Hai1 & - fahem u caqel (Le sage devin) \\
\hline Arbaoua Arb1 & - kayd eDDràr (la ruse des co-épouses) \\
\hline Tetouan Tét1 & - hina (Hina) \\
\hline Khemisset Khé1 & - rruda l mensiya (le cimetière abandonné) \\
\hline Salé Sal1 & - zbiba (Zbiba) \\
\hline Larache Lar1 & - rrajel u sebca d ewladu (L'homme et ses sept fils) \\
\hline Meknès Mek1 & - lalla saqyet $1 \Omega$ beq (L'arroseuse du basilic) \\
\hline $\begin{array}{l}\text { Moulay } \\
\text { Driss Zerhoun Zer1 }\end{array}$ & - manteq ttyur (L'oiseau-oracle) \\
\hline Sefrou Sef1 & - luRziyyel w e $\Omega$ tu (Le petit daim et sa sœur) \\
\hline Settat Set1 & - cayfa rmada (Aicha des cendres) \\
\hline
\end{tabular}

\section{NOTES}

1. Yacine 1993, p. 39.

\section{RÉSUMÉS}

Cet article traite des images de la femme dans quelques contes marocains. Il s'agit de saisir les représentations sociales véhiculées par la littérature orale et les attitudes sous-jacentes exprimées par les relations de rôles des personnages féminins dans le cadre d'une démarche de type sociolinguistique.

This article examines the portrayal of women in some Moroccan tales and stories. Its main aim is to describe, through a sociolinguistic approach, the social representations of women in oral literature and to identify the underlying attitudes expressed by the female characters and the roles they fill.

\section{AUTEUR}

\section{LEILA MESSAOUDI}

Leila MESSAOUDI, marocaine, est professeur de littérature et responsable de l'UFR « Langage et société », Département de langue et littérature françaises - Kénitra (Maroc). Elle est la coordinatrice du GREC (Groupe de recherches et d'études sur le conte) et du GREFEN (Groupe de 
recherches et d'études sur les femmes dans le Nord du Maroc). Ses plus récentes publications sont : « Note sur l'affriquée dj dans le parler des Jbala (Nord du Maroc) », Estudios de dialectologia norteafricana y andalusi, Zaragoza, Area de estudios arabes (1996) ; « Le conte marocain. Aspects sociolinguistiques de la matière racontée », Recherches pédagogiques, $n^{\circ} 6$, Meknès, Association Marocaine des Enseignants de Français (AMAEF) (1996) ; « Contes marocains du Nord-Ouest. Propositions pour une approche sociolinguistique » (à paraître). 